\title{
The macroscopic spectrum of nilmanifolds with an emphasis on the Heisenberg groups
}

\author{
Constantin Vernicos*
}

\begin{abstract}
Take a Riemannian nilmanifold, lift its metric on its universal cover. In that way one obtains a metric invariant under the action of some co-compact subgroup. We use it to define metric balls and then study the spectrum of the Dirichlet Laplacian. Using homogenization techniques we describe the asymptotic behavior of the spectrum when the radius of these balls goes to infinity. This involves the spectrum, which we call macroscopic spectrum, of a so called homogenized operator on a specific domain. Furthermore we show that the first macroscopic eigenvalue is bounded from above, by a universal constant in the case of the three dimensional Heisenberg group, and by a constant depending on the Albanese torus for the other nilmanifolds. We also show that the Heisenberg groups belong to a family of nilmanifolds, where the equality characterizes some pseudo-left-invariant metrics.
\end{abstract}

Mathematics Subject Classification (2000). Primary 53C24; Secondary 58C40, 74Q99.

Keywords. Spectrum of the Laplacian, nilmanifolds, homogenization, stable norm, asymptotic volume, Albanese metric, rigidity.

\section{Introduction and statement of the results}

This article deals with geometric properties of large balls in periodic Riemannian manifolds. A Riemannian manifold $\left(N^{n}, g\right)$ is periodic if it possesses a discrete group $\Gamma$ of isometries with a compact fundamental domain. Given $x_{0} \in N^{n}$, we are interested in the asymptotic behavior of two geometric invariants of the metric ball, $B_{g}\left(x_{0}, \rho\right)$, with radius $\rho$ and centered at $x_{0}$, as $\rho$ tends to $\infty$ :

- the Riemannian volume $\operatorname{Vol}_{g}\left(B_{g}\left(x_{0}, \rho\right)\right)$;

- the spectrum of the Dirichlet Laplacian on $B_{g}\left(x_{0}, \rho\right)$.

Our approach consists in rescaling the metric, i.e., replacing the original Riemannian metric $g$ on $N$ with $g_{\rho}=1 / \rho^{2} g$, so that $B_{g}\left(x_{0}, \rho\right)$ becomes $B_{g_{\rho}}\left(x_{0}, 1\right)$, and applying homogenization techniques to the family of Riemannian manifolds with

*Partially supported by European project ACR OFES number 00.0349 and grant of the FNRS 20-65060.01 
boundary $N_{\rho}=\left(B_{g_{\rho}}\left(x_{0}, 1\right), g_{\rho}\right)$. There are several notions of convergence of metric spaces (see [Gro81b], [Gro93]). It turns out that the balls $N_{\rho}$ converge, in the Gromov-Hausdorff sense, to a compact metric space if and only if the group $\Gamma$ contains a finite index subgroup $\Gamma^{\prime}$ that is nilpotent, torsion-free, and finitely generated. This follows from a celebrated result of M. Gromov [Gro81a], characterizing finitely generated groups of polynomial growth, completed by P. Pansu [Pan83] and Van den Dries-Wilkie [vdDW84]. Therefore, actions of nilpotent groups seem to provide the proper setting for application of homogenization techniques in Riemannian geometry. According to Malcev, such a group uniquely embeds into a simply connected nilpotent Lie group $G$, and $G / \Gamma$ is called a nilmanifold. In the sequel, we assume that $N=G$ is equipped with a $\Gamma$-invariant Riemannian metric. The manifold $N^{n}$ can be viewed as the Riemannian universal covering of $M^{n}=G / \Gamma$ equipped with the quotient metric.

Although the results presented here are geometric in nature, we use homogenization techniques. Hence this article can be read under two different lights.

1.1. From the geometric point of view: The Riemannian volume and the Dirichlet spectrum of $B_{g}\left(x_{0}, \rho\right)$ are linked by Weyl's asymptotic formula, which states that if $\lambda_{k}(\rho)$ is the $\mathrm{k}^{\text {th }}$ eigenvalue of the ball of radius $\rho$ and $\operatorname{Vol}(\rho)$ is its volume, then as $k \rightarrow \infty$, there exists a universal constant $c(n)$ such that

$$
\lambda_{k}(\rho) \sim c(n) \frac{k^{2 / n}}{\operatorname{Vol}^{2 / n}(\rho)}
$$

One could expect that the asymptotic behaviors of the volume and the Dirichlet spectrum when the radius of the balls increases would be related. This is not the case; we shall see that they are described by two different limit metrics.

Problem 1. Make the asymptotic behavior of the volume of a ball with respect to its radius, precise, and extract geometric information from it.

In the case of nilmanifolds there is a precise equivalent to the volume of balls given by P. Pansu [Pan83], which depends on the algebraic structure. Let $G^{1}=G$, and $G^{i+1}=\left[G^{i}, G\right]$; then $d_{h}=\sum_{i=1}^{\infty} \operatorname{dim} G^{i}$ is called the homogeneous dimension of $G$, and

$$
\operatorname{Vol}(\rho) \sim \operatorname{Asvol}(g) \rho^{d_{h}} .
$$

The constant $\operatorname{Asvol}(g)$ is usually called the asymptotic volume. In the particular case of tori, D. Burago and S. Ivanov [BI95] gave a lower bound on the asymptotic volume, which is achieved if and only if the metric is flat (see also [Ver04] for an alternate proof in dimension 2 using homogenization theory and [Bab91] for the first proof in dimension 2).

To the nilpotent Lie group $G$, we can associate its limit group at infinity, $G_{\infty}$, which is nilpotent and graded. Furthermore, thanks to a theorem of K. Nomizu 
[Nom54], $H_{1}(M, \mathbb{R})$ can be identified with a subspace of the Lie algebra of $G_{\infty}$, hence to a left invariant distribution $\mathscr{H}$ of vector fields over $G_{\infty}$. Thus to any norm on $H_{1}(M, \mathbb{R})$ we can associate a left-invariant sub-finslerian structure on $G_{\infty}$, hence, thanks to Chow's theorem on accessibility, a left-invariant distance on $G_{\infty}$. For a Euclidean norm we obtain a left-invariant sub-Riemannian structure. The metric $g$ induces two important norms on $H_{1}(M, \mathbb{R})$. The first one, called the stable norm, comes from the sup norm on the 1 -forms over $M$, which induces a norm (usually not Euclidean) on $H^{1}(M, \mathbb{R})$, and by duality on $H_{1}(M, \mathbb{R})$. The second one, called the Albanese metric, comes from the $L^{2}$ normalized norm on 1-forms, which induces a Euclidean norm on $H^{1}(M, \mathbb{R})$, and by duality on $H_{1}(M, \mathbb{R})$. The two distances induced by these two metrics on $G_{\infty}$ are often said to be of Carnot-Carathéodory type. We call them, respectively, the stable distance and the Albanese distance.

The following inequality, if not the best one, gives a hint of what we might expect for all nilmanifolds.

Theorem 1. Let $\left(M^{n}, g\right)$ be a nilmanifold. Let $G_{\infty}$ be the limit group at infinity associated to the universal covering of $M^{n}$. Then the asymptotic volume of $M^{n}$ satisfies the following:

1. $\operatorname{Asvol}(\mathrm{g}) \geq \operatorname{Vol}_{\mathrm{g}}\left(M^{n}\right) \frac{\mu\left(B_{\mathrm{al}}(1)\right)}{\mu\left(D_{M}\right)} ;$

2. in case of equality the stable norm coincides with the Albanese metric.

Here, $\mu$ is a Haar measure on $G_{\infty}, B_{\mathrm{al}}(1)$ is the unit ball of the Albanese distance centered at the unit element, and $D_{M}$ is the image in $G_{\infty}$ of a fundamental domain on the universal covering of $M^{n}$, by the canonical projection.

Concerning the spectrum of the Laplacian on balls, a theorem of R. Brooks [Bro85] (see also Sunada [Sun89]) states that the bottom of the spectrum on the universal cover is zero if and only if the fundamental group is amenable. The first eigenvalue goes to the bottom of the spectrum as the radius of the ball goes to infinity. R. Brooks's theorem implies, in our case, as the fundamental group is nilpotent hence amenable, that the first eigenvalue goes to zero as the radius goes to infinity.

Problem 2. Make the speed of convergence to the bottom of the spectrum on the universal cover with respect to the radius, precise, and extract more geometric information from the spectrum of large balls.

To state our results to that problem, let us remark that to the Albanese metric we can also associate a kind of Laplacian $\Delta_{\infty}$ on $G_{\infty} . \Delta_{\infty}$ is usually called the Kohn Laplacian. It is a dilation invariant hypoelliptic second order differential operator, which is symmetric and without a constant term. 
Theorem 2. Let $\left(M^{n}, g\right)$ be a nilmanifold, with universal cover $\tilde{M}$, and let $x \in \tilde{M}$. Let $B_{g}(x, \rho)$ be the corresponding Riemannian ball of radius $\rho$ and center $x \in \widetilde{M}$, and let $\lambda_{1}\left(B_{g}(x, \rho)\right)$ be the first eigenvalue of the Laplacian for the Dirichlet problem on $B_{g}(x, \rho)$. Then

1. $\lim _{\rho \rightarrow+\infty} \rho^{2} \lambda_{1}\left(B_{g}(x, \rho)\right)=\lambda_{1}^{\infty} \leq \lambda_{1}(g$, Alb $)$;

2. in case of equality, the stable norm coincides with the Albanese metric, hence all harmonic 1-forms are of constant length.

Here, $\lambda_{1}(g, A l b)$ is the first eigenvalue of the Kohn Laplacian arising from the Albanese metric for the Dirichlet problem on $B_{\mathrm{al}}(1)$, the unit ball of the Albanese distance centered at the unit element. Furthermore, for tori this is a constant dependent only on the dimension, and for the 3-dimensional Heisenberg group it is also independent of the metric.

In the case of a 2-step nilmanifold with a 1-dimensional center, we can determine for which metrics equality holds. We call these metrics pseudo-left-invariant (see Section 6 for the definition). One of their main properties being that they arise as fiber metrics over a flat torus (i.e. the nilmanifold submerges onto a flat torus).

Theorem 3. In the case of a 2-step nilmanifold whose center is one dimensional, the Albanese metric and the stable norm coincide if and only if the metric is pseudo-leftinvariant.

Actually this behavior is shared by all the eigenvalues, and Theorem 2 is partially a consequence of the following:

Theorem 4. Let $\left(M^{n}, g\right)$ be a nilmanifold, with universal cover $\tilde{M}$, and let $x \in \tilde{M}$. Let $B_{g}(x, \rho)$ be the corresponding Riemannian ball of radius $\rho$, and center $x \in \tilde{M}$ and let $\lambda_{i}\left(B_{g}(x, \rho)\right)$ be the $i^{\text {th }}$ eigenvalue of the Laplacian for the Dirichlet problem on $B_{g}(x, \rho)$.

Then there exists an hypoelliptic operator $\Delta_{\infty}$ (the Kohn Laplacian of the Albanese metric), whose $i^{\text {th }}$ eigenvalue for the Dirichlet problem on the unit ball of the stable distance (centered at the unit element) is $\lambda_{i}^{\infty}$, and such that

$$
\lim _{\rho \rightarrow \infty} \rho^{2} \lambda_{i}\left(B_{g}(\rho)\right)=\lambda_{i}^{\infty} .
$$

We call $\left(\lambda_{i}^{\infty}\right)_{i \in \mathbb{N}}$ the macroscopic spectrum.

1.2. From the point of view of analysis, let

$$
L=-\frac{\partial}{\partial x_{i}} a_{i j}(x) \frac{\partial}{\partial x_{i}},
$$


be a uniformly elliptic differential operator on $\mathbb{R}^{n}$, and assume that the coefficients $a_{i j}$ are periodic, i.e., $a_{i j}(x+k)=a_{i j}(x)$ for any $k \in \mathbb{Z}^{n}$ and $C^{\infty}$. After rescaling we get a family of operators

$$
0<\epsilon \leq 1, \quad L_{\epsilon}=-\frac{\partial}{\partial x_{i}} a_{i j}\left(\frac{x}{\epsilon}\right) \frac{\partial}{\partial x_{i}} .
$$

We can associate, to this family of operators, a so-called homogenized operator,

$$
L_{0}=-q_{i j} \frac{\partial}{\partial x_{i}} \frac{\partial}{\partial x_{j}}
$$

Now, if $\mathrm{D}$ is a domain of $\mathbb{R}^{n}$, then we can consider the Dirichlet problem for this family of operators, and hence we have eigenvalues and eigenfunctions, denoted respectively by

$$
\lambda_{0}^{\epsilon} \leq \lambda_{1}^{\epsilon} \leq \lambda_{2}^{\epsilon} \leq \cdots \quad \text { and } \quad \phi_{0}^{\epsilon}, \phi_{1}^{\epsilon}, \phi_{2}^{\epsilon}, \ldots
$$

We now have the following problem.

Problem 1'. Study the convergence of $\lambda_{i}^{\epsilon}$ and $\phi_{i}^{\epsilon}$ to $\lambda_{i}^{0}$ and $\phi_{i}^{0}$, respectively, as $\epsilon \rightarrow 0$.

In the case of $\mathbb{R}^{n}$, this is the subject of Chapter III of [OSY92], and Chapter 11 of [JKO94], and of [CD99].

The operators $L_{\epsilon}$ define Riemannian distances $d_{\epsilon}$. So another related problem is the following:

Problem 2'. Study the relationship between the distance $d_{\epsilon}$ and the distance $d_{0}$.

The papers [Dav93], [Nor94] and [Nor97] are related to this problem in conjunction with the existence of bounds on the heat kernel (see also [KS00] for a probabilistic approach).

The present paper is concerned with the problem above, when $\mathbb{R}^{n}$ is replaced by a nilpotent Lie group $N$ and $\mathbb{Z}^{n}$ by a uniform lattice $\Gamma$ of $N$. Homogenization in this context, when $N$ is stratified (graded), has been the subject of [BBJR95], [BMT96] and [BMT97].

However our paper differs in three ways from the previous work. First of all, we are not dealing with a stratified group, hence we must not only homogenize the operator, but also the space, by using its associated graded Lie group. Secondly we begin by studying a family of elliptic operators, which happens to have an hypoelliptic homogenized operator. And finally, our domain moves with the operator. The relationship with the long time asymptotics of the heat kernel is shortly studied in Section 8 . 


\section{Geometry of nilmanifolds}

2.1. Nilpotent Lie algebras. Let $\mathfrak{u}$ be a Lie algebra. One says that it is nilpotent if the sequence defined by

$$
\mathfrak{u}^{1}=\mathfrak{u}, \quad \mathfrak{u}^{i+1}=\left[\mathfrak{u}^{i}, \mathfrak{u}\right],
$$

is such that for some $k \in \mathbb{N}, \mathfrak{u}^{k+1}=\{0\}$. Let $r$ be the smallest of such $k$; then we say that $\mathfrak{u}$ is an $r$-step nilpotent Lie algebra.

A distinguished family of nilpotent Lie algebras consists of the graded ones. A nilpotent Lie Algebra $\mathfrak{u}$ is graded if it admits a decomposition:

$$
\mathfrak{u}=V_{1} \oplus \cdots \oplus V_{r}
$$

such that

1. $V_{i}$ is a complement of $\mathfrak{u}^{i+1}$ in $\mathfrak{u}^{i}$;

2. $\left[V_{i}, V_{j}\right] \subset V_{i+j}$.

It is quite important in our work that to such a graduation one can attach a oneparameter group of automorphisms $\left(\tau_{\rho}\right)_{\rho \in \mathbb{R}^{+}}$called dilations such that:

$$
\tau_{\rho}(x)=\rho^{i} x \quad \text { for all } x \in V_{i} .
$$

In fact, the existence of such a family of dilations is equivalent to the existence of a graduation. These dilations play the same role as the dilations in Euclidean space.

Not all nilpotent Lie algebras are graded. But to each nilpotent Lie algebra, we can associate a graded nilpotent one in the following way:

$$
\mathfrak{u}_{\infty}=\sum_{i=1}^{r} \mathfrak{u}_{i} / \mathfrak{u}_{i+1},
$$

the Lie bracket being induced. We will denote by $\tilde{\pi}: \mathfrak{u} \rightarrow \mathfrak{u}_{\infty}$ the induced projection and by $\tilde{\tau}_{\rho}$ the dilations in $\mathfrak{u}_{\infty}$.

The Homogeneous dimension of $\mathfrak{u}$ is the number

$$
d_{h}=\sum_{i=1}^{r} i \operatorname{dim}\left(\mathfrak{u}^{i} / \mathfrak{u}^{i+1}\right) .
$$

There is another way to make that graded Lie algebra appear: start with a nilpotent Lie algebra $\mathfrak{u}$, remark that for all $i, \mathfrak{u}^{i+1} \subset \mathfrak{u}^{i}$, and build a basis $\left(X_{i}\right)_{i}$ of $\mathfrak{u}$ by taking independent vectors $X_{d_{1}+\cdots+d_{i-1}+1}, \ldots, X_{d_{1}+\cdots+d_{i-1}+d_{i}}$ such that the vector space $V_{i}$ that they span is a complement of $\mathfrak{u}^{i+1}$ in $\mathfrak{u}^{i}$. Hence the direct sum (1) holds. We shall denote by $\operatorname{pr}_{V_{i}}$ the projection induced on $V_{i}$ by this direct sum. Now we define a function $\tau_{\rho}: \mathfrak{u} \rightarrow \mathfrak{u}$ by

$$
\tau_{\rho}\left(X_{p}\right)=\rho^{\alpha(p)} X_{p}
$$


with $\alpha(p)=i$ if $d_{i-1}<p \leq d_{i}$, and $d_{0}=0$.

We obtain a new Lie algebra $\mathfrak{u}_{\rho}$ by modifying the Lie bracket in the following way: for any $X$ and $Y$ in $\mathfrak{u}_{\rho},[X, Y]_{\rho}=\tau_{1 / \rho}\left[\tau_{\rho} X, \tau_{\rho} Y\right]$. Thus $\tau_{\rho}$ becomes a Lie algebra isomorphism from $\mathfrak{u}_{\rho}=\left(\mathfrak{u},[\cdot, \cdot]_{\rho}\right)$ to $(\mathfrak{u},[\cdot, \cdot])$.

Now as $\rho$ goes to infinity, $\mathfrak{u}_{\rho}$ goes to $\mathfrak{u}_{\infty}$, in the sense that for $i, j=1, \ldots, n$, we have

$$
\left[X_{i}, X_{j}\right]_{\infty}=\operatorname{pr}_{V_{\alpha(i)+\alpha(j)}}\left[X_{i}, X_{j}\right]
$$

Notice that all $\mathfrak{u}_{\rho}$ have the same graded Lie algebra. We will denote by $\tilde{\pi}_{\rho}$ the projection from $\mathfrak{u}_{\rho}$ to $\mathfrak{u}_{\infty}$ (in fact we could avoid the subscript in $\tilde{\pi}_{\rho}$, because we can identify the Lie algebras as linear spaces).

Notice that if the Lie algebra is graded, then $[X, Y]_{\rho}=[X, Y]$, and $\tau_{\rho}$ is a Lie algebra automorphism. Otherwise, remark that for all $X \in \mathfrak{u}_{\rho}$

$$
\tilde{\pi}\left(\tau_{\rho}(X)\right)=\tilde{\tau}_{\rho}\left(\tilde{\pi}_{\rho}(X)\right) .
$$

2.2. Remarks on exponential coordinates. Let $G$ be the simply connected Lie group associated with the nilpotent Lie algebra $\mathfrak{u}$. For nilpotent Lie groups, the exponential is a diffeomorphism between the Lie algebra and the Lie group, hence thanks to the exponential coordinates, we can identify $G$, as a differential manifold, with some $\mathbb{R}^{n}$ :

$$
\phi: \mathbb{R}^{n} \rightarrow G, \quad \phi: x=\left(x_{1}, \ldots, x_{n}\right) \mapsto \exp x_{1} X_{1} \ldots \exp x_{n} X_{n} .
$$

Let $X_{i}^{*}$ be the dual form of $X_{i}$.

Moreover, we denote by $\delta_{\rho}$ the following family of dilations:

$$
\delta_{\rho}\left(x_{1}, \ldots, x_{n}\right)=\left(\rho^{\alpha(1)} x_{1}, \ldots, \rho^{\alpha(n)}\right) .
$$

Notice also that $d \delta_{\rho}=\tau_{\rho}$. We define a family of group products $*_{\rho}$ by setting

$$
x *_{\rho} y=\delta_{1 / \rho}\left[\delta_{\rho}(x) \delta_{\rho}(y)\right] .
$$

Finally

$$
x *_{\infty} y=\lim _{\rho \rightarrow \infty} x *_{\rho} y .
$$

Thus we get a family of nilpotent Lie groups $G_{\rho}=\left(G, *_{\rho}\right), 0<\rho \leq \infty$, whose Lie algebras are isomorphic, respectively, to the algebras $\mathfrak{u}_{\rho}, 0<\rho \leq \infty$. We also denote by $\pi_{\rho}: G_{\rho} \rightarrow G_{\infty}$ the function which sends $x \in G_{\rho}$ to $x \in G_{\infty}$, i.e., $\pi_{\rho}=\phi_{\infty} \circ \phi_{\rho}^{-1}$ (and to simplify $\pi_{1}=\pi$ ).

Observe that for $1 \leq j \leq d_{1}$, the $x_{j}$ live on $G /[G, G]$.

If $e \in G$ is the unit element and $X \in \mathfrak{u}$, then for $\rho \in \overline{\mathbb{R}}, X^{\rho}$ will be the $*_{\rho}$ left invariant field in $G_{\rho}$ such that $X^{\rho}(e)=X(e)$. Thus to the basis $\left(X_{i}\right)$ defined in 2.1, we will associate the $*_{\rho}$ left invariant fields $\left(X_{i}^{\rho}\right)$. Notice also that

$$
d \delta_{\rho}\left(X_{i}^{\rho}\right)=\tau_{\rho}\left(X_{i}^{\rho}\right)=\rho^{\alpha(i)} X_{i}
$$


We also define $\nabla_{\mathscr{H}}$ by

$$
\nabla_{\mathscr{H}} f=\left(X_{1}^{\infty} \cdot f, \ldots, X_{d_{1}}^{\infty} \cdot f\right)
$$

\section{Asymptotic behavior of the distance}

\subsection{The stable norm}

3.1.a. Recall that $\left(M^{n}, g\right)$ is a manifold whose universal covering is a simply connected nilpotent Lie group $G$. We shall denote by $\tilde{g}$ the lifted metric on $G$. On the graded nilpotent Lie group $G_{\infty}$ associated to $G$, we obtain a natural distribution by left multiplication of $V_{1}=\mathfrak{u}_{1} / \mathfrak{u}_{2} \subset \mathfrak{u}_{\infty}$. We shall call that distribution horizontal and denote it by $\mathcal{H}$.

Let us remark that since the Lie algebra $\mathfrak{u}_{\infty}$ is generated by $V_{1}$, a basis of $V_{1}$ satisfies the so called Chow (or Hörmander) condition in the Lie group $G_{\infty}$. Let us recall what the stable norm is:

Definition 5. Let $\|\cdot\|_{\infty}^{*}$ be the quotient of the sup norm on 1-forms, arising from the metric $g$, on the cohomology $H^{1}\left(M^{n}, \mathbb{R}\right)$. Then its dual norm on the homology $H_{1}\left(M^{n}, \mathbb{R}\right)$, is called the stable norm and we denote it by $\|\cdot\|_{\infty}$.

By a theorem of K. Nomizu [Nom54], $H_{1}\left(M^{n}, \mathbb{R}\right) \equiv V_{1}$, thus we can transport the stable norm on $\mathcal{H}$. Now the Rashevsky-Chow theorem (see Theorem 2.4, p. 15, in [BR96]) asserts that two points of $G_{\infty}$ can be joined by a curve tangent to $\mathscr{H}$ (usually called an admissible curve). For an admissible curve $\gamma:[a, b] \rightarrow G_{\infty}$, we consider its stable length $l_{\infty}(\gamma)=\int_{a}^{b}\|\dot{\gamma}(t)\|_{\infty} d t$. Hence we can define a distance $d_{\infty}$, which we call the stable distance, between two points of $G_{\infty}$, by taking the infimum of the stable lengths of admissible curves between these points. This kind of distance is usually said to be of Carnot-Carathéodory type. We shall call the unit ball for the stable distance centered at 0 the stable ball and denote it by $B_{\infty}(1)$.

3.1.b. For any $x, y \in G_{\rho}$, let us introduce $d_{\rho}(x, y)=\frac{d_{g}\left(\delta_{\rho} x, \delta_{\rho} y\right)}{\rho}$. Then the work of P. Pansu [Pan83], implies that for any $x, y \in G_{\rho}$

$$
\lim _{\rho \rightarrow \infty} \frac{d_{\infty}\left(\pi_{\rho}(x), \pi_{\rho}(y)\right)}{d_{\rho}(x, y)}=\lim _{\rho \rightarrow \infty} \frac{d_{\infty}\left(\pi \circ \delta_{\rho}(x), \pi \circ \delta_{\rho}(y)\right)}{d_{g}\left(\delta_{\rho} x, \delta_{\rho} y\right)}=1 .
$$

This implies the simple convergence of the functionals $x \mapsto d_{\rho}\left(0, \pi_{\rho}^{-1}(x)\right)$ toward $x \mapsto d_{\infty}(0, x)$ on $B_{\infty}(1) \backslash \partial B_{\infty}(1)$. 
3.1.c. Remark that the distance $d_{\rho}$ is also given by the metric $g_{\rho}$ on $G_{\rho}$, obtained by rescaling the pull back of the metric $\tilde{g}$ on $G$ in the following way:

$$
g_{\rho}=\frac{1}{\rho^{2}}\left(\delta_{\rho}\right)^{*} \tilde{g}
$$

\subsection{Gromov-Hausdorff convergence of balls}

3.2.a. Recall that a family of spaces $X_{n}$, endowed with metrics $d_{n}$ and measures $\mu_{n}$, is said to converge in the Gromov-Hausdorff Measured sense toward $(X, d, \mu)$ if and only if there is a family $\left(f_{n}\right)_{n \in \mathbb{N}}$, where for all $n, f_{n}$ is an $\mu_{n}$ measurable function from $X_{n}$ to $X$, and there is a sequence $\left(\epsilon_{n}\right)$, decreasing to 0 , such that

1. the $\epsilon_{n}$ neighborhood of $f_{n}\left(X_{n}\right)$ in $X$ is $X$;

2. for any $x, y \in X_{n},\left|d_{n}(x, y)-d\left(f_{n}(x), f_{n}(y)\right)\right| \leq \epsilon_{n}$;

3. for any continuous function $u: X \rightarrow \mathbb{R}$ we have

$$
\int_{X_{n}} u \circ f_{n} d \mu_{n} \rightarrow \int_{X} u d \mu
$$

3.2.b. Let $\mu_{\rho}$ (resp. $\mu_{g}$ ) denote the Riemannian volume associated to $g_{\rho}$ (resp. $g$ ), and let $\mu_{\infty}$ be defined as follows. Let $D_{\Gamma}$ be a fundamental domain in $G$ and $\mu$ a Haar measure on $G_{\infty}$, then (recall that $\pi$ is the canonical projection from $G \rightarrow G_{\infty}$ )

$$
\mu_{\infty}=\frac{\mu_{g}\left(D_{\Gamma}\right)}{\mu\left(\pi\left(D_{\Gamma}\right)\right)} \mu .
$$

Adding to this that for any compact domain $A$ in $G_{\infty}$, whose boundary is of Haar measure 0 , and any function $f \in L^{1}\left(A, \mu_{\infty}\right)$, we have

$$
\lim _{\rho \rightarrow \infty} \int_{\pi_{\rho}^{-1}(A)} f\left(\pi_{\rho}(x)\right) d \mu_{\rho}(x)=\int_{A} f d \mu_{\infty} .
$$

Theorem 6. The family of metric spaces $\left(B_{\rho}(1), d_{\rho}, \mu_{\rho}\right)$ converges in the GromovHausdorff measure topology to $\left(B_{\infty}(1), d_{\infty}, \mu_{\infty}\right)$ as $\rho$ goes to infinity.

To prove the convergence (2) let $A$ be a domain in $G_{\infty}$, then $\pi_{\rho}^{-1}(A)$ belongs to $G_{\rho}$ and $\delta_{\rho} \circ \pi_{\rho}^{-1}(A)$ belongs to $G$. We will denote by $*$ the law group of $G$. Let $z_{1}, \ldots, z_{k}$ and $\zeta_{1}, \ldots, \zeta_{l}$ be elements of $\Gamma$ such that $\zeta_{j} * D_{\Gamma} \cap \delta_{\rho} \circ \pi_{\rho}^{-1}(A) \neq \emptyset$ for any $j$, and

$$
\bigcup_{i} z_{i} * D_{\Gamma} \subset \delta_{\rho} \circ \pi_{\rho}^{-1}(A) \subset \bigcup_{j} \zeta_{j} * D_{\Gamma}
$$


Let us notice that

$$
\mu_{g}\left(D_{\Gamma}\right)=\frac{\mu_{g}\left(D_{\Gamma}\right)}{\mu\left(\pi\left(D_{\Gamma}\right)\right)} \mu\left(\pi\left(D_{\Gamma}\right)\right)=\mu_{\infty}\left(\pi\left(D_{\Gamma}\right)\right) .
$$

Then we get

$$
\begin{aligned}
\sum_{i} \inf _{\delta_{\rho} \circ \pi_{\rho}^{-1}(x) \in z_{i} * D_{\Gamma}} f(x) \mu_{\infty}\left(\pi\left(D_{\Gamma}\right)\right) & \leq \int_{\delta_{\rho} \circ \pi_{\rho}^{-1}(A)} f\left(\tilde{\delta}_{1 / \rho} \circ \pi(x)\right) d \mu_{g}(x) \\
& \leq \sum_{j} \sup _{\delta_{\rho} \circ \pi_{\rho}^{-1}(x) \in \zeta_{j} * D_{\Gamma}} f(x) \mu_{\infty}\left(\pi\left(D_{\Gamma}\right)\right) .
\end{aligned}
$$

Dividing both sides by $\rho^{d_{h}}$ (see 2.1), we get:

$$
\begin{aligned}
\sum_{i} \inf _{x \in \pi_{\rho} \circ \delta_{1 / \rho}\left(z_{i} * D_{\Gamma}\right)} f(x) & \mu_{\infty}\left(\tilde{\delta}_{1 / \rho} \circ \pi\left(D_{\Gamma}\right)\right) \leq \int_{\pi_{\rho}^{-1}(A)} f\left(\pi_{\rho}(x)\right) d \mu_{\rho} \\
& \leq \sum_{j} \sup _{x \in \pi_{\rho} \circ \delta_{1 / \rho}\left(\zeta_{j} * D_{\Gamma}\right)} f(x) \mu_{\infty}\left(\tilde{\delta}_{1 / \rho} \circ \pi\left(D_{\Gamma}\right)\right) .
\end{aligned}
$$

Then the extremal terms are Riemann sums that converge toward $\int_{A} f d \mu_{\infty}$.

3.2.c. We are now able to define and identify the asymptotic volume by

$$
\operatorname{Asvol}(g)=\lim _{\rho \rightarrow \infty} \frac{\mu_{g}\left(B_{g}(\rho)\right)}{\rho^{d_{h}}}=\mu_{\infty}\left(B_{\infty}(1)\right)
$$

\subsection{Convergence of the elements of the set $\mathcal{L}^{2}$}

3.3.a. For $\rho \in \overline{\mathbb{R}}, L_{\rho}^{2}=L^{2}\left(B_{\rho}(1), d \mu_{\rho}\right)$ will be the space of square integrable functions over the ball $B_{\rho}(1)$, which is a Hilbert space with the scalar product

$$
(u, v)_{\rho}=\int_{B_{\rho}(1)} u \bar{v} d \mu_{\rho}
$$

Its norm will be denoted by $|\cdot| \rho$.

Let $\mathcal{L}^{2}$ be the set of nets $\left(u_{\rho}\right)_{\rho \in \mathbb{R}^{+}}$such that for $1 \leq \rho \leq \infty, u_{\rho} \in L_{\rho}^{2}$. Thanks to the Gromov-Hausdorff measured convergence of balls, we can give a meaning to the sentence "the net $\left(u_{\rho}\right)_{\rho \in \mathbb{R}^{+}}$converges" in the following way.

Definition 7. Let $\left(u_{\rho}\right)_{\rho \in \mathbb{R}^{+}}$be an element of $\mathcal{L}^{2}$, we say that it strongly converges to $u_{\infty}$ if and only if there exists a net $\left(v_{\alpha}\right)$ in $C^{0}\left(B_{\infty}(1)\right)$ strongly converging to $u_{\infty}$ in $L_{\infty}^{2}$, and such that

$$
\lim _{\alpha} \limsup _{\rho}\left|v_{\alpha} \circ \pi_{\rho}-u_{\rho}\right|_{\rho}=0 .
$$


This allows us to introduce the weak convergence as follows.

Definition 8. Let $\left(u_{\rho}\right)_{\rho \in \mathbb{R}^{+}}$be an element of $\mathcal{L}^{2}$. We say that it converges weakly to $u_{\infty}$, if for every strongly convergent net $\left(v_{\rho}\right)_{\rho \in \mathbb{R}^{+}}$of $\mathcal{L}^{2}$, we have

$$
\lim _{\rho \rightarrow+\infty}\left(u_{\rho}, v_{\rho}\right)_{\rho}=\left(u_{\infty}, v_{\infty}\right)_{\infty} .
$$

For the properties of these convergences see our previous work [Ver04] and [Ver01]. It suffices to say that they satisfy the usual properties of weak and strong convergence in $L^{2}$.

3.3.b. We shall say that a function $f$ is periodic with respect to $\Gamma$ (the co-compact subgroup) if for every $\gamma \in \Gamma$ and $x \in G$ we have $f(\gamma * x)=f(x)$. Thus the metric $\tilde{g}$ lifted from $M^{n}$ to $G$ is periodic with respect to $\Gamma$.

To finish this section remark, that it is not difficult to adapt the proof of the limit (2) to obtain (see [BBJR95] page 431).

Lemma 9. Let $h$ be a function that is periodic with respect to $\Gamma$ on $G$. Let $h_{\rho}$ be defined on $G_{\rho}$ by $h_{\rho}(x)=h\left(\delta_{\rho} x\right)$. Then $\left(h_{\rho}\right)_{\rho \in \mathbb{R}^{+}}$weakly converges in $\mathcal{L}^{2}$ toward

$$
h_{\infty}=\frac{1}{\mu_{g}\left(D_{\Gamma}\right)} \int_{D_{\Gamma}} h d \mu_{g} .
$$

I.e. for any $u_{\rho} \rightarrow u_{\infty}$ strongly in $\mathcal{L}^{2}$, we have

$$
\int_{B_{\rho}(1)} u_{\rho} h_{\rho} d \mu_{\rho} \rightarrow h_{\infty} \int_{B_{\infty}(1)} u_{\infty} d \mu_{\infty} .
$$

\section{Behavior of the eigenvalues: setting}

\subsection{The Albanese metric}

4.1.a. Let $D_{\Gamma}$ be a fundamental domain for the action of $\Gamma$ on $G$. Let $\chi^{i}$ be the unique solution (up to an additive constant) of

$$
\Delta \chi^{i}=\Delta x_{i} \text { on } D_{\Gamma}, \quad \text { for } 1 \leq i \leq r,
$$

that is periodic with respect to $\Gamma$.

Let us define the operator $\Delta_{\infty}$ by

$$
\begin{aligned}
\Delta_{\infty} f & =-\frac{1}{\operatorname{Vol}_{g}(M)} \sum_{1 \leq i, j \leq d_{1}}\left(\int_{D_{\Gamma}} g^{i j}-\sum_{k=1}^{n} g^{i k} X_{k} \cdot \chi^{j} d \mu_{g}\right) X_{i}^{\infty} \cdot X_{j}^{\infty} f \\
& =-\sum_{1 \leq i, j \leq d_{1}} q^{i j} X_{i}^{\infty} \cdot X_{j}^{\infty} f .
\end{aligned}
$$


Remark that $\eta_{j}(x)=\chi^{j}(x)-x_{j}$ is a harmonic function on $G$, and by construction so are the 1-forms $d \eta_{i}$ on the nilmanifold. It is not difficult to show the following.

Proposition 10. Let $\langle\cdot, \cdot\rangle_{2}$ be the scalar product induced on 1-forms by the Riemannian metric $g$. Then

$$
q^{i j}=\frac{1}{\operatorname{Vol}(g)}\left\langle d \eta_{i}, d \eta_{j}\right\rangle_{2}=q^{j i} .
$$

Thus $\Delta_{\infty}$ is an Hypoelliptic operator.

4.1.b. Recall that thanks to Nomizu's work [Nom54], $H_{1}\left(M^{n}, \mathbb{R}\right) \equiv V_{1}$, hence by duality we get that the dimension of $H^{1}\left(M^{n}, \mathbb{R}\right)$ is $d_{1}$. Remark that $\left(q^{i j}\right)$ is the matrix of the $L^{2}$ normalized scalar product on harmonic 1-forms, written in the basis $\left(d \eta_{i}\right)$, hence on $H^{1}\left(M^{n}, \mathbb{R}\right)$ by Hodge's theorem (whose norm will be written $\left.\|\cdot\|_{2}\right)$. By duality it gives a scalar product on $H_{1}\left(M^{n}, \mathbb{R}\right)$ (whose norms will be written $\|\cdot\|_{2}^{*}$ ).

The norm $\|\cdot\|_{2}^{*}$ induces another Carnot-Carathéodory metric, which we shall call the Albanese metric and denote by $d_{\mathrm{al}}$, as follows. Take on $\mathscr{H}_{e} \equiv H_{1}(M, R)$ (the horizontal subspace of the tangent space at the unit element) an orthonormal basis $Y_{1}(e), \ldots, Y_{d_{1}}(e)$ for $\|\cdot\|_{2}^{*}$. It induces a left-invariant orthonormal frame field on $\mathcal{H}$, and for any admissible curve $\gamma:[a, b] \rightarrow G_{\infty}$, we have that $\dot{\gamma}(t)=$ $\sum_{i=1}^{d_{1}} \alpha_{i}(t) Y_{i}(\gamma(t))$. Then the Albanese length of $\gamma$ is $l_{\mathrm{al}}(\gamma)=\int_{a}^{b}\left(\sum_{i=1}^{d_{1}} \alpha_{i}^{2}(t)\right)^{1 / 2} d t$, and the Albanese distance between two points is the shortest Albanese length among all admissible curves joining them. A comparison of the $L^{2}$ norm and the $L^{\infty}$ norm gives the following

Proposition 11. For every 1 -form $\alpha$ and $\gamma \in H_{1}\left(M^{n}, \mathbb{R}\right)$ we have

$$
\|\alpha\|_{2} \leq\|\alpha\|_{\infty}^{*} \text { and }\|\gamma\|_{\infty} \leq\|\gamma\|_{2}^{*} .
$$

In other words the unit ball $B_{\mathrm{al}}(1)$ of the Albanese metric $d_{\mathrm{al}}$ is included in $B_{\infty}(1)$.

Proof. For $\alpha$ a 1-form we have

$$
\|\alpha\|_{2}=\left(\frac{1}{\operatorname{Vol}_{g}(M)} \int_{M}|\alpha|^{2} d \mu_{g}\right)^{1 / 2} \leq \sup _{x \in M}|\alpha(x)|=\|\alpha\|_{\infty}^{*} .
$$

Hence our proposition follows, first by passing to the quotient and by duality, and finally by integrating over admissible paths.

4.2. The eigenvalues, at last! All the balls considered here, will be centered at a fixed point $x_{0}$ of the universal covering of $M^{n}=(G / \Gamma, g)$. We study the eigenvalues of the Dirichlet problem on $B_{g}(\rho)$, the geodesic ball of radius $\rho$ :

$$
\begin{cases}\Delta \phi=\lambda \phi & \text { on } B_{g}(\rho) \\ \phi=0 & \text { on } \partial B_{g}(\rho)\end{cases}
$$


It is well known that the eigenvalues are a discrete family accumulating at infinity. We shall denote them by $\lambda_{1}(\rho) \leq \lambda_{2}(\rho) \leq \cdots \leq \lambda_{i}(\rho) \ldots$.

R. Brooks's theorem [Bro85] on the first eigenvalue of the whole group implies that as $\rho$ goes to infinity, $\lambda_{1}(\rho) \rightarrow 0$. We are going to estimate how fast it converges in our case.

4.2.a. On each $G_{\rho}$ (see 3.1.c), we pulled back the lifted metric of $M^{n}$ on $G, \tilde{g}$, and rescaled it in the following way

$$
g_{\rho}=\frac{1}{\rho^{2}}\left(\delta_{\rho}\right)^{*} \tilde{g} .
$$

This gives a net of Riemannian manifolds $\left(G_{\rho}, g_{\rho}\right)_{\rho \in \mathbb{R}^{+}}$. Let $B_{\rho}(1)$ be the unit geodesic ball for the metric $g_{\rho}$, and consider the Dirichlet problem for $\Delta_{\rho}$ the Laplacian associated to $g_{\rho}$, i.e.,

$$
\begin{cases}\Delta_{\rho} \phi=\psi & \text { on } B_{\rho}(1) \\ \phi=0 & \text { on } \partial B_{\rho}(1)\end{cases}
$$

To a function $f$ from $B_{g}(\rho)$ to $\mathbb{R}$ let us associate a function $f_{\rho}$ on $B_{\rho}(1)$ by $f_{\rho}(x)=f\left(\delta_{\rho} \cdot x\right)$. Then it is an easy calculation to see that for any $x \in B_{\rho}(1)$,

$$
\rho^{2}(\Delta f)\left(\delta_{\rho} \cdot x\right)=\left(\Delta_{\rho} f_{\rho}\right)(x) .
$$

This implies that the eigenvalues of $\Delta_{\rho}$ on $B_{\rho}(1)$ are exactly the eigenvalues of $\Delta$ on $B_{g}(\rho)$ multiplied by $\rho^{2}$.

Enlightened by what happens on tori we would like to show that the net of resolvents of the Laplacians $\left(\Delta_{\rho}\right)_{\rho \in \mathbb{R}^{+}}$compactly converges towards the resolvent of $\Delta_{\infty}$, which implies the convergence of the spectrum towards the spectrum of $\Delta_{\infty}$ for the Dirichlet problem on $B_{\infty}(1)$ (see Theorem 15, 17 and 21 of [Ver04]).

4.3. Upper bound on the eigenvalues, lower bound on the asymptotic volume and the equality cases. Recall that $B_{\mathrm{al}}(1)$ is the unit ball for the Albanese metric on $G_{\infty}$, centered at the unit element. Let $D$ be a bounded domain of $G_{\infty}$, and denote by $\lambda_{i}^{\infty}(D)$ the $i^{\text {th }}$ eigenvalue of $\Delta_{\infty}$ on $D$ for the Dirichlet problem. Then by Proposition 11, we have $B_{\infty}(1) \supset B_{\mathrm{al}}(1)$. Thus by the min-max property, for any $i$, we obtain

$$
\lambda_{i}^{\infty}\left(B_{\infty}(1)\right) \leq \lambda_{i}^{\infty}\left(B_{\mathrm{al}}(1)\right) .
$$

Following the maximum principle (see J.-M. Bony [Bon69]), equality holds if and only if the two balls coincide, and thus the norms in Proposition 11 also coincide. The same argument also shows that we have equality in the following estimate if and only if the stable norm and the Albanese metric coincide. 
Proposition 12. Let $\left(M^{n}, g\right)$ be a nilmanifold. Let $G_{\infty}$ be the limit group at infinity associated to the universal covering of $M^{n}$. Then the asymptotic volume of $M^{n}$ satisfies the following inequality:

$$
\operatorname{Asvol}(\mathrm{g}) \geq \mu_{\mathrm{g}}\left(M^{n}\right) \frac{\mu\left(B_{\mathrm{al}}(1)\right)}{\mu\left(\pi\left(D_{\Gamma}\right)\right)} .
$$

Here, $\mu$ is a Haar measure on $G_{\infty}, B_{\mathrm{al}}(1)$ is the unit ball for the Albanese distance and $D_{\Gamma}$ a fundamental domain on the universal covering of $M^{n}$.

Proof. From 11, for any Haar measure $\mu$, one gets the following inequality:

$$
\mu\left(B_{\mathrm{al}}(1)\right) \leq \mu\left(B_{\infty}(1)\right) .
$$

We can conclude by taking the Haar measure $\mu_{\infty}$ for $\mu$ (see Section 3.2), giving the asymptotic volume.

\section{Homogenization and proof of Theorem 4}

The first step consists in showing the convergence of the metric geodesic balls with respect to the Gromov-Hausdorff measure topology (completed in 3.2).

\subsection{Asymptotic compactness}

5.1.a. Let us now define the various functional spaces involved. Recall (see 3.3.a) that for $\rho \in \overline{\mathbb{R}}^{+}, L_{\rho}^{2}=L^{2}\left(B_{\rho}(1), d \mu_{\rho}\right)$ is the Hilbert space of square integrable functions over the ball $B_{\rho}(1)$ with the norm $|\cdot|_{\rho}$.

5.1.b. Following the usual nomenclature, we will be interested in the following spaces, for an $r$-step nilmanifold (see Section 2.1):

$$
\begin{gathered}
H_{\rho}^{1}\left(B_{\rho}(1)\right)=\left\{v \mid v, X_{i}^{\rho} \cdot v \in L^{2}\left(B_{\rho}(1), d \mu_{\rho}\right), 1 \leq \alpha(i) \leq r\right\} \\
\text { (resp. } \left.H_{\infty}^{1}\left(B_{\infty}(1)\right)=\left\{v \mid v, X_{i}^{\infty} \cdot v \in L^{2}\left(B_{\infty}(1), d \mu_{\infty}\right), 1 \leq i \leq d_{1}\right\}\right) .
\end{gathered}
$$

These spaces become Hilbert spaces when endowed with the quadratic forms $\|\cdot\|_{\rho}$, defined by

$$
\begin{gathered}
\|v\|_{\rho}^{2}=|v|_{\rho}^{2}+\sum_{1 \leq \alpha(i) \leq r}\left\|X_{i}^{\rho} \cdot v\right\|_{\rho}^{2} \\
\text { (resp. } \left.\|v\|_{\infty}^{2}=|v|_{\infty}^{2}+\sum_{1 \leq i \leq d_{1}}\left\|X_{i}^{\infty} \cdot v\right\|_{\infty}^{2}\right) .
\end{gathered}
$$


We will denote by $H_{\rho, 0}^{1}\left(B_{\rho}(1)\right)$ the closure in $H_{\rho}^{1}\left(B_{\rho}(1)\right)$, with respect to the norm $\|\cdot\|_{\rho}$, of the space of $C^{\infty}\left(B_{\rho}(1)\right)$ functions with compact support in $B_{\rho}(1)$.

5.1.c. We can define a self adjoint operator on $L_{\rho}^{2}$, whose resolvent will be $R_{\lambda}^{\rho}$ for $\lambda \in \mathbb{R}$, thanks to the Friedrichs extension of the Laplacian (sub-Laplacian for $\left.\Delta_{\infty}\right)$ defined on $H_{\rho, 0}^{1}\left(B_{\rho}(1)\right)$, endowed with the following quadratic form

$$
\|v\|_{\rho, 0}^{2}=|v|_{\rho}^{2}+\left(v, \Delta_{\rho} v\right)_{\rho} .
$$

Now for a bounded net in $\left(H_{\rho, 0}^{1}\left(B_{\rho}(1)\right)\right)_{\rho \in \mathbb{R}^{+}}$with respect to these quadratic forms we have the following Lemma.

Lemma 13. Let $\left(u_{\rho}\right)_{\rho \in \mathbb{R}^{+}}$be a net with $u_{\rho} \in H_{\rho, 0}^{1}\left(B_{\rho}(1)\right)$ for every $\rho \geq 1$, and assume the existence of a constant $C$ such that for every $\rho \geq 1$, we have

$$
\left\|u_{\rho}\right\|_{\rho, 0} \leq C
$$

Then there is sub-net which is strongly convergent in $\mathcal{L}^{2}$.

Proof. Let $B$ be a compact set such that $\bigcup_{\rho \in \mathbb{R}^{+}} \pi_{\rho}\left(B_{\rho}(1)\right) \subset B \subset G_{\infty}$. We are going to show that the strong convergence in $L^{2}\left(B, \mu_{\infty}\right)$ implies the strong convergence in $\mathcal{L}^{2}$. Then the compact embedding of $H_{\infty}^{1}(B)$ in $L^{2}\left(B, \mu_{\infty}\right)$ will conclude the proof.

Let us first notice that the periodicity with respect to $\Gamma$, and the co-compactness of $\Gamma$ gives the existence of two constants $\alpha$ and $\beta$ such that (we suppose the norms are defined on $B$, and identify $B$ and $\pi_{\rho}^{-1} B$ )

$$
\alpha|v|_{\infty} \leq|v|_{\rho} \leq \beta|v|_{\infty} .
$$

Let us start by taking a net $\left(v_{\rho}\right)$, strongly converging in $L^{2}\left(B, \mu_{\infty}\right)$ to $v_{\infty}$. We also assume $v_{\rho} \circ \pi_{\rho} \in H_{\rho, 0}^{1}\left(B_{\rho}(1)\right)$ for every $\rho$ and is zero outside $B_{\rho}(1)$ (because it is all we need).

First we will prove that $v_{\infty} \in L_{\infty}^{2}$ (we mean that, outside $B_{\infty}(1), v_{\infty}$ can be considered equal to zero), indeed, the strong $L^{2}$ convergence implies the existence of a subnet of $\left(v_{\rho}\right)$ which simply converges almost every where to $v_{\infty}$. Hence the GromovHausdorff convergence implies that $v_{\infty}$ is zero almost everywhere on $B \backslash B_{\infty}$.

Thus, let us take $c_{p} \in C_{0}^{\infty}\left(B_{\infty}(1)\right), p \in \mathbb{N}$, such that $\left(c_{p}\right)_{p \in \mathbb{N}}$ is a sequence of functions strongly converging to $v_{\infty}$ in $L_{\infty}^{2}$. We have

$$
\left|c_{p} \circ \pi_{\rho}-v_{\rho} \circ \pi_{\rho}\right|_{\rho} \leq \beta\left|c_{p}-v_{\infty}\right|_{\infty}+\gamma\left|v_{\infty}-v_{\rho}\right|_{\infty} .
$$

Now let $\varepsilon>0$. Then for $p$ large enough, $\beta\left|c_{p}-v_{\infty}\right|_{\infty} \leq \varepsilon$. We fix $p$ large enough, and take $\rho$ large enough for the second term to converge to 0 , which gives us the strong convergence we needed (see Definition 7). 
Now to conclude, observe that from the assumptions, the net $\left(u_{\rho} \circ \pi_{\rho}^{-1}\right)$ (if need be we extend this function by zero outside $\left.B_{\rho}(1)\right)$ is bounded in $H_{\infty}^{1}(B)$, hence using the compact embedding of $H_{\infty}^{1}(B)$ in $L^{2}\left(B, \mu_{\infty}\right)$ (with the right regularity assumption on the boundary of $B$ ), we can extract a strongly converging net in $L^{2}\left(B, \mu_{\infty}\right)$ and by what we just did in $\mathcal{L}^{2}$.

5.2. Compact convergence of the resolvents. For $\lambda>0$ and $\rho>1$, let $a_{\lambda}^{\rho}(u, v)=$ $\left(\Delta_{\rho} u, v\right)_{\rho}+\lambda(u, v)_{\rho}$ and $G_{\lambda}^{\rho}$ be the operator from $L_{\rho}^{2}$ to $H_{\rho, 0}^{1} \subset L_{\rho}^{2}$ such that

$$
a_{\lambda}^{\rho}\left(G_{\lambda}^{\rho} f, \phi\right)=(f, \phi)_{\rho} \quad \text { for all } \phi \in H_{\rho, 0}^{1} .
$$

For any $u, v \in H_{\infty, 0}^{1}$, let

$$
a_{\lambda}^{\infty}(u, v)=\int_{B_{\infty}(1)} q^{i j} X_{i}^{\infty} u X_{j}^{\infty} v d \mu_{\infty}+\lambda(u, v)_{\infty} .
$$

Then we define $G_{\lambda}: L_{\infty}^{2} \rightarrow H_{\infty, 0}^{1}$, by

$$
a_{\lambda}^{\infty}\left(G_{\lambda} F, \Phi\right)=(F, \Phi)_{\infty} \quad \text { for all } \Phi \in H_{\infty, 0}^{1}
$$

The aim of this part is the following theorem, after noticing that $R_{\lambda}^{\rho}=-G_{-\lambda}^{\rho}$ and $R_{\lambda}^{\infty}=-G_{-\lambda}$.

Theorem 14. For every $\lambda<0$, the net of resolvents $\left(R_{\lambda}^{\rho}\right)_{\rho \in \mathbb{R}^{+}}$of the net of Laplacians $\left(\Delta_{\rho}\right)_{\rho \in \mathbb{R}^{+}}$converges compactly to $R_{\lambda}^{\infty}$, the resolvent of $\Delta_{\infty}$ from the homogenized problem, i.e, for any net $\left(u_{\rho}\right)_{\rho \in \mathbb{R}^{+}}$of $\mathcal{L}^{2}$ weakly converging, the net $\left(R_{\lambda}^{\rho} \cdot u_{\rho}\right)_{\rho \in \mathbb{R}^{+}}$ of $\mathcal{L}^{2}$ strongly converges to $R_{\lambda}^{\infty} \cdot u_{\infty}$.

The proof is an adaptation of Tartar's method of oscillating test functions (see Chapter 8 of [CD99] for the classical method).

Proof. First step: Let $f_{\rho}$ be a weakly convergent net to $f$ in $\mathcal{L}^{2}$. Then up to subnets

$$
\begin{aligned}
& u_{\rho}=G_{\lambda}^{\rho} f_{\rho} \rightarrow \tilde{u}_{\lambda} \text { strongly in } \mathcal{L}^{2} ; \\
& P_{\rho}=\left(g_{\rho}^{i j}\right) \nabla G_{\lambda}^{\rho} f_{\rho} \rightarrow \tilde{P}_{\lambda} \text { weakly in } \mathcal{L}^{2} \text {. }
\end{aligned}
$$

One obtains (13) because the net $\left(f_{\rho}\right)_{\rho \in \mathbb{R}^{+}}$is uniformly bounded in $\mathcal{L}^{2}$, and for all $\rho \in \mathbb{R}, f_{\rho}$ is also bounded in $H_{\rho}^{-1}$, the dual space of $H_{\rho, 0}^{1}$. Thus thanks to equality (11) and Lemma 13 , we can extract a strongly converging net in $\mathcal{L}^{2}$ from the uniformly bounded net $\left(G_{\lambda}^{\rho} f_{\rho}\right)_{\rho \in \mathbb{R}^{+}}$(with respect to the norms $\left(\|\cdot\|_{\rho, 0}\right)_{\rho \in \mathbb{R}^{+}}$). To get (14), simply remark that $\left(P_{\rho}\right)_{\rho \in \mathbb{R}^{+}}$is also bounded in $\mathcal{L}^{2}$. 
Now for any $\phi_{\infty} \in L_{\infty}^{2}$, by passing to the limit in equation (11), we obtain

$$
\int_{B_{\infty}(1)} \tilde{P}_{\lambda} \cdot \nabla_{\mathscr{H}} \phi_{\infty} d \mu_{\infty}+\lambda\left(u_{\lambda}^{*}, \phi_{\infty}\right)_{\infty}=\left(f, \phi_{\infty}\right)_{\infty} .
$$

Before passing to the next step, remark that $\tilde{P}_{\lambda}$ is horizontal. Indeed denoting by $P_{\rho}^{i}$ and $P_{\lambda}^{i}$ the coordinates of $P_{\rho}$ and $\tilde{P}_{\lambda}$, we have

$$
P_{\rho}^{i}=\left(g_{\rho}^{i j}\right) \nabla G_{\lambda}^{\rho} f_{\rho}=\rho^{2-\alpha(i)-\alpha(j)}\left(g^{i j}\left(\delta_{\rho} x\right)\right) \nabla G_{\lambda}^{\rho} f_{\rho} .
$$

So if $\alpha(i) \geq 2$, then this net of coordinates strongly converges to 0 in $\mathcal{L}^{2}$, because $\left(g^{i j}\left(\delta_{\rho} x\right)\right) \nabla G_{\lambda}^{\rho} f_{\rho}$ is also bounded for any $\rho \in \mathbb{R}^{+}$.

Second step: This step consists in showing $\tilde{P}_{\lambda}=\left(q^{i j}\right) \nabla_{\mathscr{H}} \tilde{u}_{\lambda}$ on $B_{\infty}(1)$, as it induces $\tilde{u}_{\lambda}=G_{\lambda} f$.

We just give the ingredient needed to copy the classical proof (see also [Ver04], section 4.3).

Consider $\chi^{k}(y)$ (see 4.1.a) such that its mean value on a fundamental domain is zero, and for every $k=1, \ldots, d_{1}$, define the oscillating function

$$
w_{\rho}^{k}(x)=x_{k}-\frac{1}{\rho} \chi^{k}\left(\delta_{\rho} x\right) .
$$

Then we have

$$
w_{\rho}^{k} \rightarrow x_{k} \quad \text { strongly in } \mathcal{L}^{2} .
$$

Using the usual trick in Tartar's method, we obtain for every $\varphi \in C_{0}^{\infty}\left(B_{\infty}(1)\right)$ and for $\rho$ large enough, for the support of $\varphi$ to be in $\pi_{\rho}\left(B_{\rho}(1)\right)$ :

$$
\begin{aligned}
\int_{B_{\rho}(1)} & g_{\rho}^{i j}\left(X_{j}^{\rho} u_{\rho}\left(X_{i}^{\rho}\left(\varphi \circ \pi_{\rho}\right)\right) w_{\rho}-X_{j}^{\rho} w_{\rho}\left(X_{i}^{\rho}\left(\varphi \circ \pi_{\rho}\right)\right) u_{\rho}\right) d \mu_{\rho} \\
& =\int_{B_{\rho}(1)} f_{\rho} w_{\rho} \varphi \circ \pi_{\rho} d \mu_{\rho}-\lambda \int_{B_{\rho}(1)} \varphi \circ \pi_{\rho} u_{\rho} w_{\rho} d \mu_{\rho} .
\end{aligned}
$$

To pass to the limit in this identity, we use the following facts:

Fact 1 . $\left(X_{i}^{\rho}\left(\varphi \circ \pi_{\rho}\right)\right) w_{\rho}^{k}$ strongly converges to $\left(X_{i}^{\infty} \varphi\right) x_{k}$ in $\mathcal{L}^{2}$ because, writing the left multiplication by $x$ in $G_{\rho}$ as $l_{x}^{\rho}$, we have

$$
X_{i}^{\rho}\left(\varphi \circ \pi_{\rho}\right)_{\mid x}=d \varphi_{\pi_{\rho} \circ l_{x}^{\rho}(e)} \circ d \pi_{\rho \mid l_{x}^{\rho}(e)} \circ d l_{x}^{\rho} \cdot X_{i}^{\rho}(e) .
$$

Now by definition $l_{x}^{\rho} \rightarrow l_{x}^{\infty}$ and $\pi_{\rho} \rightarrow \operatorname{id}_{G_{\infty}}$, which explains why

$$
X_{i}^{\rho}\left(\varphi \circ \pi_{\rho}\right) \rightarrow X_{i}^{\infty} \varphi
$$

pointwise (and weakly $\mathcal{L}^{2}$ from the claim in the proof of Section 3.2). 
Fact 2. For $1 \leq i, j \leq d_{1}, g_{\rho}^{i j} X_{i}^{\rho} w_{\rho}^{k}$ is periodic with respect to $\delta_{1 / \rho} \Gamma$ and weakly converges in $\mathcal{L}^{2}$, by Lemma 9 , towards its mean value

$$
q^{j k}=\frac{1}{\mu_{g}\left(D_{\Gamma}\right)} \int_{D_{\Gamma}}\left(g^{i j}(y)\left(\delta_{i k}-X_{i} \chi^{k}(y)\right)\right) d \mu_{g} .
$$

Fact 3. For $\alpha(i)+\alpha(j)>2, g_{\rho}^{i j} X_{i}^{\rho} w_{\rho}^{k}=\rho^{2-\alpha(i)-\alpha(j)} g^{i j}\left(\delta_{\rho} x\right) X_{i}^{\rho} w_{\rho}^{k}$, thus this term weakly converges in $\mathcal{L}^{2}$ towards 0 .

Hence the identity (18) becomes

$$
\int_{B_{\infty}(1)}\left(\tilde{P}_{\lambda}^{j} x_{k}-q^{j k} \tilde{u}_{\lambda}\right) X_{j}^{\infty} \varphi d \mu_{\infty}=\int_{B_{\infty}(1)} f x_{k} \varphi d \mu_{\infty}-\lambda \int_{B_{\infty}(1)} \varphi \tilde{u}_{\lambda} x_{k} d \mu_{\infty} .
$$

Furthermore, if we put $\phi_{\infty}=\varphi x_{k}$ into equation (15) and subtract the result from the equality (19), then we obtain the following identity in terms of distribution.

$$
-\sum_{j=1}^{d_{1}} X_{j}^{\infty}\left(\tilde{P}_{\lambda}^{j} x_{k}-q^{j k} \tilde{u}_{\lambda}\right)=-\sum_{j=1}^{d_{1}} X_{j}^{\infty} \tilde{P}_{\lambda}^{j} x_{k} \Longleftrightarrow \tilde{P}_{\lambda}^{k}=\sum_{j=1}^{d_{1}} q^{j k} X_{j}^{\infty} \tilde{u}_{\lambda} .
$$

5.3. Conclusion. Theorem 14 gives the compact convergence of the resolvents. Hence we can use Theorem 21 in [Ver04], which states that if the resolvents are compact, and they converge compactly, then the net of $k^{\text {th }}$ eigenvalues converges to the $k^{\text {th }}$ eigenvalue of the limit operator.

\section{Emphasis on the Heisenberg Groups in the equality case}

The aim of this part is to characterize metrics for which the inequality (6) is an equality (see also Theorem 2) for a class of nilmanifolds that contains the Heisenberg nilmanifolds. The first thing to remark, which is always true, is that equality holds if and only if the stable norm and the Albanese metric are equal. In that case, all harmonic 1-forms are of constant pointwise norm (same proof as in [Ver04]). Now let us introduce the pseudo-left-invariant metrics.

Definition 15. Let $N^{n+1}=\Gamma \backslash G$ be a nilmanifold such that $G$ is 2-step nilpotent with one dimensional kernel. Let $p$ be a submersion of $N^{n+1}$ onto a flat torus $\mathbb{T}^{n}$. Let $\left(\alpha_{1}, \ldots, \alpha_{n}\right)$ be the lift of an orthonormal basis of harmonic 1-forms over the torus, and choose a 1-form $\vartheta$ of $N^{n+1}$ such that $d \vartheta=p^{*} b$, where $b$ is a closed 2-form 
over the torus (in other words we chose a connection). Let $g_{\vartheta}$ be the Riemannian metric such that the dual basis of $\left(\alpha_{1}, \ldots, \alpha_{n}, \vartheta\right)$ is orthonormal. Thus $p$ becomes a Riemannian submersion. We will call such a metric pseudo-left-invariant or bundlelike.

The idea is that if the 2 -form $b$ has constant coefficients, then $\vartheta$ can be chosen so that the above construction gives a left invariant metric. Thus this pseudo-leftinvariant metric can be seen as a perturbation of a left invariant metric, obtained by perturbing a left invariant basis of vector fields.

We are now able to give our precise claim.

Lemma 16. Let $\left(\mathbb{H}_{2 n+1}, g\right)$ be the $2 n+1$-dimensional Heisenberg group, equipped with a periodic metric. Then its stable norm coincides with its Albanese metric if and only if $g$ is pseudo-left-invariant.

Remark also that in the case of the 3-dimensional Heisenberg group, the function $\lambda_{1}(\mathrm{~g}, \mathrm{Alb})$ in Theorem 2 is actually a constant that does not depend on the metric. This is due to the fact that, up to isometries, there is only one left-invariant sub-Riemannian metric in that case (see chapter IV of [Ver01]). Hence in that case, the theorem has a similar form as the theorem for tori (see [Ver04]), for which the function is also constant because up to isometries there is only one Euclidean metric on $\mathbb{R}^{n}$.

Actually, we have a result that is slightly more general than Lemma 16. We focus on 2-step nilmanifolds, whose Lie algebras have a 1-dimensional center.

Lemma 17. Let $\left(M^{n+1}, g\right)$ be a 2-step nilmanifold whose center is of dimension 1 . Then its stable norm and its Albanese metric coincide if and only if the metric is pseudo-left-invariant.

As the Albanese metric and the stable norm coincide if and only if all harmonic 1 -forms are of constant norm, Lemma 17 is a consequence of the main theorem in [NV04]:

Theorem 18 (P.-A. Nagy, C. Vernicos [NV04]). Let $\left(M^{n+1}, g\right)$ be a Riemannian manifold with first Betti number equal to $n$, all of whose harmonic 1-forms are of constant norm. Then $\left(M^{n+1}, g\right)$ is a 2-step nilmanifold whose center is of dimension 1 , and $g$ is pseudo-left-invariant.

\section{Graded nilmanifolds with totally geodesic fibers over a torus}

There is one last particular case we would like to study, the case where the nilmanifold is graded (i.e. its algebra is nilpotent and graded as defined in Section 2.1), and the 
metric on $\left(M^{n}, g\right)$ is as follows. We suppose that the first Betti number $b_{1}\left(M^{n}\right)=k$, and we recall that $\mathscr{H}$ is the horizontal distribution coming from $V_{1}$ (see Sections 3.1.a and 2.1). Moreover we assume that we have the following Riemannian submersion, with totally geodesics fibers and with a metric equivariant on the fibers:

$$
[M, M] \hookrightarrow\left(M^{n}, g\right) \stackrel{p}{\longrightarrow}\left(\mathbb{T}^{k}, \check{g}\right),
$$

where $d p_{x}$ is an isometry (we write $\left.\hat{g}=g_{\mid \mathcal{H}}\right)$ from $\left(\mathscr{H}_{x}, \hat{g}_{x}\right)$ to $\left(T_{p(x)} \mathbb{T}^{k}, \check{g}_{p(x)}\right)$.

Then, in the case of equality in Theorems 1 and 2, the Albanese map is a Riemannian submersion, which implies that $\breve{g}$ is flat. Which in turn, using our assumptions implies that the metric $g$ is left invariant (indeed see Chapter 9 Section F in [Bes87]). In other words:

Proposition 19. Let $(M, g)$ satisfy the above assumptions. The Albanese metric and the stable norm coincides if and only if the metric is left invariant.

In other words, we could say heuristically that for sub-Riemannian metrics the equality case in Theorem 2 (which holds in that context too, see [Ver01] for the convergence of the spectrum) characterizes the left-invariant sub-Riemannian metrics.

\section{On the long time asymptotics of the heat kernel}

Let $(G / \Gamma, g)$ be a nilmanifold and $(G, \tilde{g})$ its universal cover with the lifted metrics. Recall that we associated to this Lie group the net $\left(G_{\rho}, g_{\rho}\right)$ of Riemannian manifolds. Let us focus on the heat kernel:

$$
\left\{\begin{array}{l}
\left.\frac{\partial u}{\partial t}+\Delta u=0 \quad \text { in }\right] 0,+\infty[\times G ; \\
u(0, x)=u_{o}(x) .
\end{array}\right.
$$

Let us introduce the rescaled functions on $G_{\rho}$,

$$
u_{\rho}(t, x)=\rho^{d_{h}} u\left(\rho^{2} t, \delta_{\rho} x\right), \quad \rho>0 .
$$

Then an easy computation shows that $u$ is a solution of (20) if and only if $u_{\rho}$ is a solution of

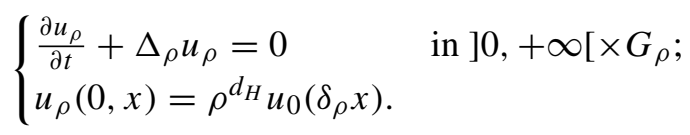

Thus the study of $u(t, \cdot)$ as $t$ goes to infinity is related to the study of $u_{\rho}(1, \cdot)$ as $\rho \rightarrow \infty$. We can imitate the proof of Theorem 14 to obtain:

Theorem 20. The net of resolvent $\left(R_{\lambda}^{\rho}\right)$ weakly converges to the resolvent $\left(R_{\lambda}^{\infty}\right)$ of $\Delta_{\infty}$ on $G_{\infty}$. 
Imitating the proof of Theorems 4 and 6 in [ZKON79], as in [BBJR95], we get the following theorem (let $d_{\mathrm{al}}(e, x)=|x|$ be the Albanese distance between the unit element and $x$ ).

Theorem 21. The fundamental solution $k(t, x, y)$ of (20) has the following asymptotic expansion

$$
k(t, x, y)=k_{\infty}(t, \pi(x), \pi(y))+t^{-\frac{d_{h}}{2}} \theta(t, x, y) .
$$

Here $k_{\infty}(t, x, y)$ is the fundamental solution of

$$
\left.\frac{\partial u_{\infty}}{\partial t}+\Delta_{\infty} u_{\infty}=0 \text { in }\right] 0,+\infty\left[\times G_{\infty},\right.
$$

and $\theta(t, x, y) \rightarrow 0$ uniformly as $t \rightarrow \infty$ on $|x|^{2}+|y|^{2} \leq$ at, for any fixed constant $a>0$.

The next theorem follows by integrating the previous one.

Theorem 22. Let $u_{0} \in L^{1}(G) \cap L^{\infty}(G)$. Then $u(t, x)$, the solution of (20), has the following asymptotic expansion:

$$
u(t, x)=c_{0} t^{-\frac{d_{h}}{2}} \int_{G} u_{0}(y) d y+t^{-\frac{d_{h}}{2}} \theta(t, x),
$$

and $\theta(t, x)$ converges uniformly to 0 for $|x|<R$, where $R$ is a positive constant, and $c_{0}$ depends on $\Delta_{\infty}$.

Acknowledgments. Many thanks to the referees for their careful reading and improvements. Thanks to F. Newberger the cats seats, and the dog stands!

\section{References}

[Ale02] G. K. Alexopoulos, Sub-Laplacians with Drift on Lie Groups of Polynomial Volume Growth. Mem. Amer. Math. Soc. 155, Amer. Math. Soc., Providence, RI, 2002. Zbl 0994.22006 MR 1878341

[Bab91] I. K. Babenko, Volume rigidity of two-dimensional manifolds. Math. Notes 48 (1-2) (1991), 629-632. Zbl 0717.53029 MR 1081887

[BBJR95] CH. J. K. Batty, 0. Bratteli, P. E. T. Jorgensen, and D. W. Robinson, Asymptotics of periodic subelliptic operators. J. Geom. Anal. 5 (4) (1995), 427-443. Zbl 0861.43005 MR 1393089

[BD98] A. Braides and A. Defranceschi, Homogenization of Multiple Integrals. Oxford Lecture Ser. Math. Appl. 12, The Clarendon Press, Oxford University Press, New York 1998. Zbl 0911.49010 MR 1684713 
[Bes87] A. L. Besse, Einstein Manifolds. Ergeb. Math. Grenzgeb. (3) 10, Springer-Verlag, Berlin 1987. Zbl 0613.53001 MR 0867684

[BI95] D. Burago and S. Ivanov, On asymptotic volume of tori. Geom. Funct. Anal 5 (5) (1995), 800-808. Zbl 0846.53043 MR 1354290

[BLP78] A. Bensoussan, J.-L. Lions, and G. Papanicolaou, Asymptotic analysis for periodic structures. Stud. Math. Appl. 5, North-Holland Publishing Co., Amsterdam-New York 1978. Zbl 0404.35001 MR 0503330

[BMT96] M. Biroli, U. Mosco, and N. A. Tchou, Homogenization for degenerate operators with periodical coefficients with respect to the Heisenberg group. C. R. Acad. Sci. Paris Ser. I 322 (1996), 439-444. Zbl 0851.47046 MR 1381780

[BMT97] M. Biroli, U. Mosco, and N. A. Tchou, Homogenization by the Heisenberg group. Adv. Math. 7 (1997), 809-837. Zbl 0889.35009 MR 1476278

[Bon69] J.-M. Bony, Principe du maximum, inégalite de Harnack et unicité du problème de Cauchy pour les opérateurs elliptiques dégénérés. Ann. Inst. Fourier (Grenoble) 19 (1) (1969), 277-304, xii. Zbl 0176.09703 MR 0262881

[BR96] A. Bellaïche and J.-J. Risler (eds.), Sub-Riemannian Geometry. Progr. Math. 144 Birkhäuser, Basel 1996. Zbl 0848.00020 MR 1421821

[Bro85] R. Brooks, The bottom of the spectrum of a Riemannian covering. J. Reine Angew. Math. 357 (1985), 101-114. Zbl 0553.53027 MR 0783536

[CD99] D. Cioranescu and P. Donato, An Introduction to Homogenization. Oxford Lecture Ser. Math. Appl. 17, Oxford University Press, New York 1999. Zbl 0939.35001 MR 1765047

[Dan91] D. Danielli, A compact embedding theorem for a class of degenerate Sobolev space. Rend. Sem. Mat. Univ. Politec. Torino 49 (1991), 339-420. Zbl 0789.46026 MR 1231060

[Dav93] E. B. Davies, Heat kernels in one dimension. Quart. J. Math. Ser. (2) 44 (175) (1993), 283-299. Zbl 0830.34019 MR 1240472

[FSC97] B. Franchi, R. Serapioni, and F. Serra Cassano, Approximation and imbedding theorems for weighted Sobolev spaces associated with Lipschitz continuous vector fields. Boll. Un. Mat. Ital. B(7) 11 (1997), 83-117. Zbl 0952.49010 MR 1448000

[Gro81a] M. Gromov, Groups of polynomial growth and expanding maps. Inst. Hautes Études Sci. Publ. Math. 53 (1981), 53-73. Zbl 0474.20018 MR 0623534

[Gro81b] M. Gromov, Structures métriques pour les variétés riemanniennes. Textes Mathématiques 1, CEDIC, Paris 1981. Zbl 0509.53034 MR 0682063

[Gro93] M. Gromov, Asymptotic invariants of infinite groups. In Geometric group theory, Vol. 2. London Math. Soc. Lecture Note Ser. 182, Cambridge University Press, Cambridge 1993, 1-295. Zbl 0841.20039 MR 1253544

[JKO94] V. V. Jikov, S. M. Kozlov, and O. A. Oleiŭnik, Homogenization of differential operators and integral functionals. Springer-Verlag, Berlin 1994. Zbl 0838.35001 MR 1329546

[KS00] M. Kotani and T. Sunada,. Albanese maps and off diagonal long time asymptotics for the heat kernel. Commun. Math. Phys. 209 (2000), 633-670. Zbl 0953.58022 MR 1743611 
[Lot99] J. Lott, Remark about heat diffusion on periodic spaces. Proc. Amer. Math. Soc. 127 (4) (1999), 1243-1249. Zbl 0949.58027 MR 1476376

[Mas93] G. Dal Maso, An introduction to $\Gamma$-convergence. Progr. Nonlinear Differential Equations Appl. 8, Birkhäuser, Boston, MA, 1993. Zbl 0816.49001 MR 1201152

[Mat51] Y. Matsushima, On the discrete subgroups and homogeneous spaces of nilpotent lie groups. Nagoya J. Math. 2 (1951), 95-110. Zbl 0045.31002 MR 0041144

[Nom54] K. Nomizu, On the cohomology of compact homogeneous spaces of nilpotent Lie groups. Annals of Math. (2) 59 (1954), 531-538. Zbl 0058.02202 MR 0064057

[Nor94] J. R. Norris, Heat kernel bounds and homogenization of elliptic operators. Bull. London Math. Soc 26 (1) (1994), 75-87. Zbl 0790.35008 MR 1246475

[Nor97] J. R. Norris, Long-time behaviour of heat flow: global estimates and exact asymptotics. Arch. Rational Mech. Anal. 140 (2) (1997), 161-195. Zbl 0899.35015 MR 1482931

[NV04] P.-A. Nagy and C. Vernicos, The length of harmonic forms on a compact Riemannian manifold. Trans. Amer. Math. Soc. 356 (6) (2004), 2501-2513. Zbl 1048.53023 MR 2048527

[OSY92] O. A. Oleı̆nik, A. S. Shamaev, and G. A. Yosifian, Mathematical problems in elasticity and homogenization. Stud. Math. Appl. 26, North-Holland Publishing Co., Amsterdam 1992. Zbl 0768.73003 MR 1195131

[Pan83] P. Pansu, Croissance des boules et des géodésiques fermées dans les nilvariétés. Ergodic Theory Dynam. Systems 3 (1983), 415-445. Zbl 0509.53040 MR 0741395

[Pan99] P. Pansu, Profil isopérimétrique, métriques périodiques et formes d'équilibre des cristaux. ESAIM Control Optim. Calc. Var. 4 (1999), 631-665 (electronic). Zbl 0939.53022 MR 1746171

[Sun89] T. Sunada, Unitary representations of fundamental groups and the spectrum of twisted Laplacians. Topology 28(2) (1989), 125-132.Zbl 0681.53025 MR 1003577

[vdDW84] L. van den Dries and A. J. Wilkie, Gromov's theorem on groups of polynomial growth and elementary logic. J. Algebra 89 (2) (1984), 349-374. Zbl 0552.20017 MR 0751150

[Ver01] C. Vernicos, Spectre asymptotiques des nilvariétés graduées. Thèse de doctorat, Université Grenoble I, Joseph Fourier, 2001.

[Ver04] C. Vernicos, The macroscopic sound of tori. Pacific J. Math. 213 (1) (2004), 121-156. Zbl 02101147 MR 2040254

[ZKON79] V. V. Žhikov, S. M. Kozlov, O. A. Oleı̆nik, and Kha T’en Ngoan, Averaging and gconvergence of differential operators. Russian Math. Surveys 34 (5) (1979), 69-147. Zbl 0445.35096 MR 0562800

Received October 24, 2002; revised May 28, 2003

Constantin Vernicos, Université de Neuchâtel, Institut de Mathématiques, Emile Argand 11, 2007 Neuchâtel Switzerland

E-mail: constantin.vernicos@unine.ch 